\title{
EL CHEQUE NO SE DESNATURALIZA
}

\author{
Alberto Viada Lozano \\ Profesor de Derecho Comercial \\ Universidad Católica del Norte \\ Sede Coquimbo
}

Aparece reiteradamente en la jurisprudencia de nuestros tribunales la afirmación de que, al girarse un cheque "en garantía", se estaría "desnaturalizando" dicho título de crédito y, por ende, el girador no podría haber cometido el delito de giro doloso de cheque, por haber dejado de ser cheque tal documento.

Constituyen los argumentos básicos de esta conclusión, en primer lugar el texto del inciso primero del artículo 11 del Decreto con Fuerza de Ley 707 de 1982, que fijó el Texto Refundido, Coordinado y Sistematizado de la Ley Sobre Cuentas Corrientes Bancarias y Cheques, cuyo tenor es el siguiente: "El cheque puede ser girado en pago de obligaciones o en comisión de cobranza", por lo que, al ser girado "en garantía" se estaría desnaturalizando tal documento. En segundo término se argumenta que, conforme a lo dispuesto en el artículo 13 del cuerpo legal citado, el cheque debe expresar, entre otras menciones, "el lugar y la fecha de expedición" y "la cantidad girada, en letras y números", lo que no ocurriría en los cheques "en garantía", en que la fecha o el monto, o ambas cosas, quedarían en blanco para ser puestas dichas menciones cuando se quisiera hacer efectiva la "garantía".

En mi opinión, tales actuaciones del girador no desnaturalizan el cheque, sino que son meros incumplimientos de las obligaciones que le impone el contrato de cuenta corriente bancaria, de las que devienen las responsabilidades civiles y penales que la propia ley establece, entre las cuales está precisamente el quedar afecto a las sanciones penales por la comisión del delito de giro doloso de cheque cuando, al ser presentado a su cobro, dicho documento no es pagado por el banco librado por algunas de las razones señaladas en el artículo 22 del D.F.L. 707, y, puesto el correspondiente protesto en su conocimiento mediante notificación judicial, no consignare fondos suficientes para atender al pago del cheque, de los intereses corrientes y de las costas judiciales.

Para fundamentar esta opinión, a lo primero que hay que atender es al concepto de "desnaturalización", no definido en la ley, por lo que recurrimos al Diccionario de la Real Academia de la Lengua Española, que la define como: "Acción y efecto de desnaturalizar o desnaturalizarse", y este verbo, en su segunda acepción, como: "Variar la forma, propiedades o condiciones de una cosa; desfigurarla, pervertirla".

Del mismo modo, debe quedar claro qué es un "cheque", para saber si esta intención de giro varía su forma, propiedades o condiciones; lo desfigura o pervierte. Como el cheque si está definido por la ley, aplicando la frase final del artículo 20 del Código Civil, debemos atenernos al concepto legal, establecido en el artículo 10 del D.F.L. 707 ya citado, que dispone lo siguiente: "El cheque es una orden escrita y girada 
contra un Banco para que éste pague, a su presentación, el todo o parte de los fondos que el librador pueda disponer en cuenta corriente". A ello cabe agregar lo dispuesto en el artículo 15, en el sentido que "el cheque será girado en formularios numerados que suministrará gratuitamente el librado, en talonarios de serie especial para cada librador...". Como el tenor literal de estas disposiciones es meridiano, no podemos desatenderlo.

Por último, debemos considerar el concepto de "garantía", para establecer si ello permite desnaturalizar un cheque. El Diccionario la define así: "Acción y efecto de afianzar lo estipulado"; "2. Fianza, prenda"; "3. Cosa que asegura y protege contra algún riesgo o necesidad". Ello hace evidente que "garantía", en el ámbito jurídico, es sinónimo de "caución", que está definida en el artículo 45 del Código Civil en los siguientes términos: "Caución significa generalmente cualquiera obligación que se contrae para la seguridad de otra obligación propia o ajena". Por consiguiente, debemos entender que un cheque "en garantía" es el que se gira para la seguridad del cumplimiento o pago de una obligación, propia o ajena.

Veamos ahora en qué consiste esa caución para cuyo fin se gira el cheque en estudio. En primer lugar debe aclararse que no nos estamos refiriendo al cheque "endosado en garantía" o "en prenda", puesto que tal figura jurídica no ha sido discutida y resulta de la aplicación de las normas sobre tal endoso que, para la letras de cambio, establecen los artículos 17, 21, 30 y 79 de la ley 18.092, aplicables al cheque por expresa disposición del inciso tercero del articulo 11 del D.F.L. 707. Solamente estamos tratando de dilucidar lo referente al cheque "girado en garantía. Y, para saber en qué consiste la garantía de un cheque girado con tal fin, tenemos primero que referirnos al contrato de cuenta corriente bancaria, que está definido por el artículo $1^{\circ}$ del D.F.L. 707 en los siguientes términos: "La cuenta corriente bancaria es un contrato a virtud del cual un Banco se obliga a cumplir las órdenes de pago de otra persona hasta la concurrencia de las cantidades de dinero que hubiere depositado en ella o del crédito que se haya estipulado". De más está recordar que "las órdenes de pago", a que se refiere esta definición son los cheques que en el mismo cuerpo legal se establecen en su articulo 10, ya transcrito. Debemos agregar que, conforme al artículo $3^{\circ}$, el Banco puede permitir que su comitente gire en exceso del monto del crédito estipulado o de su haber en efectivo. Para el cumplimiento de la obligación de pago del Banco, éste suministra al girador formularios numerados, en talonarios de serie especial para cada librador.

Entonces, puede afirmarse que la "seguridad del cumplimiento de la obligación" consiste precisamente en entregar una orden para que un tercero ajeno a la convención, el Banco, normalmente una sociedad anónima solvente, pague a su presentación el valor del documento, imputando para ello el dinero o crédito que tenga disponible el girador, e incluso aunque su monto exceda de su disponibilidad. Y si el Banco se niega a pagar, quedan subsistentes para el tenedor del título las acciones, civiles o penales, que emanan precisamente de la naturaleza del cheque. En otras palabras, se asegura la obligación porque es un Banco el que se obliga al pago, desde el momento que entrega el talonario de cheques, y porque, de no producirse el pago, quedan subsistentes las acciones propias del titulo de crédito contra su suscriptor. Parafraseando esta intención, el girador manifiesta: "Para asegurar el cumplimiento de tal obligación, entrego este cheque girado por mí para que en su oportunidad lo pague el Banco, o en caso contrario responderé civil o penalmente conforme a la ley". 
$Y$ es de este concepto de "seguridad" del cumplimiento de una obligación que nace el primer argumento para concluir que el giro "en garantía" no desnaturaliza el cheque. Sabemos que, conforme a lo dispuesto en el articulo 1.562 del Código Civil, el sentido en que una estipulación puede producir algún efecto deberá preferirse a aquél en que no sea capaz de producir efecto alguno. $Y$ si una persona gira, y su contraparte en el contrato recibe, un cheque para asegurar el pago de una obligación, es porque ambas partes entienden que dicho documento, título de crédito, es un cheque al momento de girarse y conserva permanentemente tal calidad. Puesto que, si al girarse el cheque con la intención de asegurar el pago de una obligación, por ese solo hecho el documento dejara de ser cheque, se desnaturalizara, nos enfrentaríamos al absurdo de que el documento no aseguraría nada, pues su tenedor carecería de todas las acciones derivadas del cheque, sirviendo el documento solamente de un principio de prueba por escrito para acreditar la existencia de la obligación garantizada. Ni siquiera tendría la eficacia de un contrato, por ser su giro un acto jurídico unilateral, y nadie puede afirmar que un contrato escrito sea garantía de las obligaciones en él contenidas, sino que es solamente un instrumento de prueba preconstituído para acreditar las convenciones de los contratantes. Debe concluirse, entonces, que el cheque girado con tal intención sigue siendo un cheque, manteniendo su portador todas las acciones que emanan de este título de crédito.

Otro argumento deriva del carácter abstracto del título de crédito que es el cheque, y de su autonomía. El inciso final del artículo 10 del D.F.L. 707 reconoce que "el cheque puede ser a la orden, al portador o nominativo". Por consiguiente, salvo que se girare nominativamente, el cheque puede circular libremente, sea mediante el endoso si es a la orden o por su simple entrega si es al portador. $Y$ en virtud de esta libre circulación, su tenedor legítimo tiene un vínculo jurídico directo con el suscriptor del título de crédito y con el Banco librado que entregó el formulario numerado y seriado especialmente, sin que tenga importancia la obligación causal o relación fundamental que lo generó, la que no consta en el cheque y, si constara, se tendría por no escrita (inc. octavo Art. 13 D.F.L. 707). De este modo, el tercero ajeno a la intención de garantía, es tenedor legítimo de un cheque, título de crédito abstracto que le permite exigir del banco librado su pago a su presentación o, en caso de no pago, exigir las responsabilidades civiles y penales de su girador. Y si este título de crédito tiene plena eficacia como cheque respecto del tercero, legítimo tenedor conforme a su ley de circulación, no existe ninguna razón para que no la tenga respecto del primer tenedor o beneficiario y del girador y el librado.

También cabe argumentar aplicando lo que señala el inciso final del artículo 1.564 del Código Civil, que permite interpretar las convenciones "por la aplicación práctica que hayan hecho de ellas ambas partes, o una de las partes con aprobación de la otra". Ello porque en la gran mayoría de los casos en que se han girado cheques "en garantía", cuando éstos han sido presentados a los Bancos, ellos los han pagado. O sea, el librado sigue considerando al documento como una orden de pago con cargo a los créditos o dinero efectivo que el girador tenga disponibles en su cuenta corriente. No se concibe, entonces, si los propios obligados consideran cheque al girado en garantía y lo pagan, que por la simple circunstancia de que no se pague, por carecer de fondos u otro motivo, el mismo cheque deba considerarse desnaturalizado y pierda toda su eficacia jurídica. 
En cuanto a la argumentación de que el inciso primero del articulo 11 del D.F.L. 707 únicamente permite girar los cheques en pago de obligaciones o en comisión de cobranza, por lo que el girado "en garantía" desvirtuaría su naturaleza, cabe señalar que en ninguna parte de esa disposición se emplea la palabra "sólo" u otra similar. siendo su redacción de carácter facultativo: "puede ser girado...". Y el mismo cuerpo legal, en el inciso séptimo del artículo 13, clarifica que, omitidas las palabras "para mí", el cheque "se entenderá girado en pago de obligaciones o estipulaciones equivalentes". De este modo, puede considerarse una "estipulación equivalente" al pago de obligaciones, cuando se "asegura" dicho pago con el giro del cheque, esto es, cuando se gira "en garantía", razón por la cual no podría considerarse esta intención como una forma de desnaturalizar el cheque.

Ahora bien, respecto a las objeciones formales relativas a la falta de fecha o de la cantidad girada, debe recordarse en primer lugar que no todo cheque girado en garantía carece de estas menciones al momento de su giro, sino que precisamente se giran por la cantidad cuyo pago se garantiza y con una fecha, aunque la mayoría de las veces esa fecha es futura. Pero la posibilidad formal de que se coloque una fecha futura está expresamente reconocida en el D.F.L. 707, que en el inciso segundo del artículo 10. después de reafirmar que "el cheque es siempre pagadero a la vista", agrega la siguiente frase final: "El cheque presentado al cobro antes del día indicado como fecha de emisión, es pagadero el día de la presentación". O sea, reconoce la ley la posibilidad de que el cheque contenga una fecha distinta de aquella en que fue girado, y no por ello deja de ser cheque, sino que expresamente dispone que "es pagadero a su presentación".

El problema se suscita cuando alguna de estas menciones, fecha o cantidad, o ambas, no se estampan en el cheque al momento de girarse, sino cuando se pretende hacer efectiva la garantía y cobrarlo al Banco. Al respecto, conviene recordar la presunción legal de que, si no es girado expresamente en comisión de cobranza, esto es, si se omitieren las palabras "para mí", el cheque "se entenderá girado en pago de obligaciones o estipulaciones equivalentes" (inc. $7^{\circ}$ Art. 13 del D.F.L. 707). Y el cheque girado en garantía no contiene las palabras "para mî", razón por la cual se presume girado en pago de obligaciones o estipulaciones equivalentes, o sea, que valen lo mismo para todos los efectos legales. Pues bien, el inciso tercero del artículo 11 del citado D.F.L. 707, es perentorio al disponer: "El cheque dado en pago se sujetará a las reglas generales de la letra de cambio, salvo lo dispuesto en la presente ley". Como este D.F.L. no contiene ninguna disposición relativa a los efectos de la omisión de menciones en el cheque, salvo la omisión de las palabras "para mî", ya vista, y la del lugar de giro (en cuyo caso se le presume extendido en la plaza en que funciona la oficina sobre la cual fue girado), debe aplicarse en lo demás las disposiciones generales de la letra de cambio.

Entre ellas, obviamente, está la del artículo $2^{\circ}$ de la ley 18.092 , que señala que el documento que no cumpla con todas las exigencias, en este caso de los artículo 13 y 15 del D.F.L. 707, no valdrá como cheque, que constituye el argumento legal más fuerte en pro de la desnaturalización del cheque en garantía, pero sólo si se hubieran omitido al momento de girarse alguna de las menciones obligatorias. Pero este argumento deja de tener asidero ante lo preceptuado en el artículo 11 de la misma ley 18.092, el que, reemplazando las referencias propias de la letra de cambio por las del cheque, dispone lo siguiente: "Sin perjuicio de lo dispuesto en el artículo $2^{\circ}$, si el cheque no contiene las 
menciones de que trata el artículo 13 del D.F.L. 707, cualquier tenedor legítimo podrá incorporarlas antes del cobro del documento, sujetándose en todo ello a las instrucciones que haya recibido de los obligados al pago del cheque". Resulta, en consecuencia, que es absolutamente legítimo para el tenedor del cheque en garantía incorporarle las menciones que se hayan omitido, incluso fecha y cantidad, siempre que lo haga con anterioridad a su presentación al cobro, sin que por ello el cheque se desnaturalice.

Es cierto que, cuando el legítimo tenedor abusare de esta facultad legal y se apartare de las instrucciones, el obligado al pago del cheque podrá eximirse del pago, pero no porque el cheque hubiere dejado de ser tal, sino porque él probó la circunstancia de que completaron las menciones contrariando sus instrucciones, e incluso tal facultad de eximirse no puede hacerla valer respecto del tenedor de buena fe. Por su parte, quien abusó del giro en blanco del cheque deberá responder penalmente, pero sin que el documento deje de ser cheque.

Resumiendo, tampoco la circunstancia de omitirse al momento del giro del cheque en garantía menciones esenciales como la fecha o la cantidad, permite desnaturalizarlo, puesto que tales menciones puede incorporarlas cualquier tenedor legítimo antes de presentarlo a su cobro, para lo que está expresamente facultado por la ley.

Además de todas las razones dadas en apoyo de la conclusión de que el cheque no se desnaturaliza, aunque se gire con la intención de asegurar el cumplimiento de una obligación, es necesario considerar que la propia ley se ha preocupado de impedir dicha desnaturalización. En efecto, un documento con apariencia de tal solamente carecería de valor como cheque si no fuera girado en los formularios numerados contenidos en talonarios de serie especial para cada librador, que proporciona el Banco librado, o si, al presentarlo a su cobro carece de alguna de las menciones esenciales establecidas en el artículo 13 del D.F.L. 707, pero, reitero, ello debe ocurrir al momento de ser presentado el documento al Banco para su cobro, porque, como ya se expuso precedentemente, aunque se omitiera alguna de ellas al momento del giro, cualquier tenedor legítimo podrá incorporar válidamente tales menciones antes del cobro del cheque. Y planteo que la propia ley se preocupa de impedir la desnaturalizaci6n del cheque porque, tras señalar en el artículo 13 las menciones que debe contener para que valga como tal y aclarar en el inciso séptimo que, si se omitieren las palabras "para mi", se entenderá girado en pago de obligaciones o estipulaciones equivalentes, dispone en el inciso octavo: "Cualesquiera otras circunstancias o cláusulas que se agregaren al cheque, se tendrán por no escritas". O sea, aunque expresamente se estampare en el cheque que fue girado en garantía o algo similar, dicha mención se tendrá por no escrita, quedando el documento conteniendo solamente las menciones que la ley exige para su validez, y ninguna otra. A lo que cabe agregar que el № 10 del artículo $3^{\circ}$ del Código de Comercio, que establece actos de comercio "formales", dispone expresamente que, entre otros, son actos de comercio las operaciones sobre cheques, "cualesquiera que sean su causa y objeto y las personas que en ella intervengan". Lo que confirma que todo documento que sea formalmente un cheque, quedará sometido a la legislación mercantil, especialmente la precisamente dictada sobre cuentas corrientes bancarias y cheques, cualquiera que sea la intención del librador al girarlo, o del tenedor al recibirlo.

Por consiguiente, no cabe sino concluir que todo cheque, girado en formulario numerado y seriado especialmente, proporcionado por el Banco librado, que contenga todas las menciones que señala el artículo 13 del D.F.L. 707 al momento de ser presen- 
tado al cobro al Banco, es y será siempre un cheque, con todas las consecuencias jurídicas propias de este titulo de crédito, especialmente las responsabilidades civiles y penales que de él emanan, sin que pueda considerarse desnaturalizado porque se haya girado en garantía, es decir, para asegurar el cumplimiento o pago de una obligación.

Por último, antes de concluir este estudio y en atención a que últimamente resulta muy significativo que, según sus obligados al pago, la mayoría de los cheques que llegan a los tribunales habrían sido girados "en garantía", resulta conveniente analizar brevemente si efectivamente ese giro tiene la característica alegada por quienes quieren eludir sus responsabilidades, o realmente se giran en pago de obligaciones. No creo que ofrezcan mayor dificultad los casos en que el monto de la obligación está determinado al momento del giro, como las compras con cheques a 30, 60, 90 días o más; o cuando se ha recibido dinero en mutuo y se gira el cheque para cumplir con la restitución en la época acordada; o si se gira el cheque por el monto de una multa o pena determinada, para el evento de no cumplir una obligación de hacer dentro de un plazo convenido, y, en general, repito, cuando el monto de la obligación está determinado desde el principio. En todos estos casos no cabe ninguna duda que el cheque se gira en pago de obligaciones, aunque la presentación al cobro ante el Banco librado esté diferida. Solamente cuando dicho monto no está plenamente establecido al momento de suscribirse el cheque seria más difícil establecer el objetivo del giro. Por ejemplo, que es una situación de ordinaria ocurrencia, cuando un comerciante encarga a su proveedor una serie de mercaderías y envía un cheque en blanco, generalmente a fecha, para pagar el precio total de las que realmente le sean remitidas, dependiendo el monto de la disponibilidad de productos para la venta que tenga el proveedor y eventualmente de las fluctuaciones de precio del mercado; o el caso de una cuenta corriente mercantil, en que el cheque se gira para pagar el saldo en contra que resultare, $u$ otros en que, repito, el monto de la obligación no estuviera determinado al momento del giro. En todos estos casos, considero que el cheque también es girado en pago de obligaciones, si bien su monto será determinado posteriormente, por lo que la cantidad quedará en blanco para ser incorporada válidamente por su tenedor legítimo conforme a lo acordado, antes de ser presentado el cheque a su cobro al Banco. Se puede objetar que al girarse el cheque en el caso de la compraventa del ejemplo no existiría obligación que pagar, pues no se habría perfeccionado el contrato de compraventa, pero bien sabemos que pueden ser objeto de una declaración de voluntad las cosas que se espera que existan y la cantidad puede ser incierta, con tal que el acto o contrato fije reglas o contenga datos que sirvan para determinarla (Art. 1461 del C. Civil); que la venta de cosas que se espera que existan se entiende hecha bajo la condición de existir; que la determinación del precio puede hacerse por cualquiera indicación o medio que lo fijen, o estipularse el "corriente de plaza" al día de la entrega u otra estipulación, incluso puede dejarse su determinación al arbitrio de un tercero (Arts. $1.808,1809$ y 1.813 del C. Civil). Por ello, resulta totalmente ajustado a derecho el giro de un cheque en pago de una obligación cuyo monto podrá determinarse ulteriormente, conforme a lo convenido, no siendo de hecho un giro en garantía.

Por todo lo expuesto, no sólo debe concluirse que el cheque girado en garantía no se desnaturaliza por tal circunstancia, sino que en la realidad tal giro no tiene la calidad que se le atribuye, sino que el cheque se gira en pago de obligaciones. 